Korean J. Math. 21 (2013), No. 4, pp. 483-493

http://dx.doi.org/10.11568/kjm.2013.21.4.483

\title{
SINGULAR POTENTIAL BIHARMONIC PROBLEM
}

\author{
Tacksun Jung And Q-Heung ChOI*
}

\begin{abstract}
We investigate the multiplicity of the solutions for a class of the system of the biharmonic equations with some singular potential nonlinearity. We obtain a theorem which shows the existence of the nontrivial weak solution for a class of the system of the biharmonic equations with singular potential nonlinearity and Dirichlet boundary condition. We obtain this result by using variational method and the generalized mountain pass theorem.
\end{abstract}

\section{Introduction}

Let $\Omega$ be a bounded domain in $R^{n}$ with smooth boundary $\partial \Omega$. Let $c \in$ $R$ and $D$ be an open subset in $R^{n}$ with compact complement $C=R^{n} \backslash D$, $n \geq 2$. Let $G$ be a $C^{2}$ function defined on $\Omega \times D$ and $u=\left(u_{1}, \ldots, u_{n}\right)$. In this paper we investigate the multiplicity of the solutions for a class of the system of the nonlinear biharmonic equations with Dirichlet boundary condition:

Received July 30, 2013. Revised December 9, 2013. Accepted December 9, 2013. 2010 Mathematics Subject Classification: 35J48.

Key words and phrases: Class of the biharmonic system, singular potential nonlinearity, Dirichlet boundary condition, variational method, generalized mountain pass theorem, (P.S. $)_{c}$ condition.

* Corresponding author.

* This work was supported by Inha University Research Grant.

(C) The Kangwon-Kyungki Mathematical Society, 2013.

This is an Open Access article distributed under the terms of the Creative commons Attribution Non-Commercial License (http://creativecommons.org/licenses/by -nc/3.0/) which permits unrestricted non-commercial use, distribution and reproduction in any medium, provided the original work is properly cited. 


$$
\begin{array}{cc}
\Delta^{2} u_{1}(x)+c \Delta u_{1}(x)=\frac{\partial}{\partial u_{1}} G(x, u(x)) & \text { in } \Omega, \\
\Delta^{2} u_{2}(x)+c \Delta u_{2}(x)=\frac{\partial}{\partial u_{2}} G(x, u(x)) & \text { in } \Omega, \\
\vdots \quad \vdots \quad \vdots & \\
\Delta^{2} u_{n}(x)+c \Delta u_{n}(x)=\frac{\partial}{\partial u_{n}} G(x, u(x)) \quad \text { in } \Omega, \\
u_{1}=\cdots=u_{n}=0, \quad \Delta u_{1}=\cdots=\Delta u_{n}=0 \quad \text { on } \partial \Omega,
\end{array}
$$

where $\operatorname{grad}_{u} G(x, u(x))=\left(G_{u_{1}}(x, u), \ldots, G_{u_{n}}(x, u)\right)$. We assume that $G \in C^{2}$ satisfies the following conditions:

$(G 1)$ There exists $R_{0}>0$ such that

$$
\sup \left\{|G(x, u)|+\left\|\operatorname{grad}_{u} G(x, u)\right\|_{R^{n}} \mid(x, u) \in \Omega \times\left(R^{n} \backslash B_{R_{0}}\right)\right\}<+\infty .
$$

(G2) There is a neighborhood $U$ of $C$ in $R^{n}$ such that

$$
G(x, u) \geq \frac{A}{d^{2}(u, C)} \text { for }(x, u) \in \Omega \times U,
$$

where $d(u, C)$ is the distance function to $C$ and $A>0$ is a constant. The system (1.1) can be rewritten as

$$
\begin{aligned}
& \Delta^{2} u(x)+c \Delta u(x)=\operatorname{grad}_{u} G(x, u(x)) \quad \text { in } \quad \Omega, \\
& u=(0, \cdots, 0), \quad \Delta u=(0, \cdots, 0) \quad \text { on } \quad \partial \Omega .
\end{aligned}
$$

Let $\lambda_{j}, j \geq 1$, be the eigenvalues and $\phi_{j}, j \geq 1$, be the corresponding eigenfunctions suitably normalized with respect to $L^{2}(\Omega)$ inner product and each eigenvalue $\lambda_{j}$ is repeated as often as its multiplicity, of the eigenvalue problem $\Delta u+\lambda u=0$ in $\Omega, u=0$ on $\partial \Omega$. The eigenvalue problem

$$
\begin{array}{cc}
\Delta^{2} u+c \Delta u=\Lambda u & \text { in } \Omega, \\
u=0, \quad \Delta u=0 \quad \text { on } \partial \Omega,
\end{array}
$$

has also infinitely many eigenvalues $\Lambda_{j}=\lambda_{j}\left(\lambda_{j}-c\right), j \geq 1$, and corresponding eigenfunctions $\psi_{j}, j \geq 1$. We note that $\Lambda_{1}<\Lambda_{2} \leq \Lambda_{3} \ldots$, $\Lambda_{j} \rightarrow+\infty$.

Our main result is the following:

Theorem 1.1. Assume that $\lambda_{j}<c<\lambda_{j+1}, j \geq 1$, and the nonlinear term $G \in C^{2}$ satisfies the conditions $(G 1)-(G 2)$. Then the system (1.1) has at least one nontrivial weak solution. 
For the proof of Theorem 1.1 we approach the variational method and use the critical point theory. In Section 2, we introduce a Banach space and the associated functional $I$ of (1.1), and recall the generalized mountain pass theorem. In Section 3, we prove that $I$ satisfies the geometric assumptions of the generalized mountain pass theorem and prove Theorem 1.1.

\section{Banach space spanned by eigenfunctions and associated functional}

Let $L^{2}(\Omega)$ be a square integrable function space defined on $\Omega$. Any element $u$ in $L^{2}(\Omega)$ can be written as

$$
u=\sum h_{k} \psi_{k} \quad \text { with } \sum h_{k}^{2}<\infty .
$$

We define a subspace $E$ of $L^{2}(\Omega)$ as follows

$$
E=\left\{u \in L^{2}(\Omega)\left|\sum\right| \Lambda_{k} \mid h_{k}^{2}<\infty\right\} .
$$

Then this is a complete normed space with a norm

$$
\|u\|=\left[\sum\left|\Lambda_{k}\right| h_{k}^{2}\right]^{\frac{1}{2}}
$$

Since $\lambda_{k} \rightarrow+\infty$ and $c$ is fixed, we have

(i) $\Delta^{2} u+c \Delta u \in E$ implies $u \in E$.

(ii) $\|u\| \geq C\|u\|_{L^{2}(\Omega)}$, for some $C>0$.

(iii) $\|u\|_{L^{2}(\Omega)}=0$ if and only if $\|u\|=0$, which is proved in [2].

Let

$$
\begin{aligned}
& E^{+}=\left\{u \in E \mid h_{k}=0 \text { if } \Lambda_{k}<0\right\}, \\
& E^{-}=\left\{u \in E \mid h_{k}=0 \text { if } \Lambda_{k}>0\right\} .
\end{aligned}
$$

Then $E=E^{-} \oplus E^{+}$, for $u \in E, u=u^{-}+u^{+} \in E^{-} \oplus E^{+}$. Let $H$ be the $n$ cartesian product space of $E$, i.e.,

$$
H=E \times E \times \ldots \times E .
$$

Let $H^{+}$and $H^{-}$be the subspaces on which the functional

$$
u \mapsto Q(u)=\int_{\Omega}\left[\|\Delta u(x)\|_{R^{n}}^{2}-c\|\nabla u(x)\|_{R^{n}}^{2}\right] d x, \quad u=\left(u_{1}, \ldots, u_{n}\right)
$$

is positive definite and negative definite, respectively. Then

$$
H=H^{+} \oplus H^{-} \text {. }
$$


Let $P^{+}$be the projection from $H$ onto $H^{+}$and $P^{-}$the projection from $H$ onto $H^{-}$. The norm in $H$ is given by

$$
\|u\|^{2}=\left\|P^{+} u\right\|^{2}+\left\|P^{-} u\right\|^{2}, \quad u=\left(u_{1}, \ldots, u_{n}\right)
$$

where $\left\|P^{+} u\right\|^{2}=\sum_{i=1}^{n}\left\|P^{+} u_{i}\right\|^{2},\left\|P^{-} u\right\|^{2}=\sum_{i=1}^{n}\left\|P^{-} u_{i}\right\|^{2}$, $u=\left(u_{1}, \ldots, u_{n}\right)$.

In this paper we are trying to find the weak solutions $u \in C^{2}(\Omega, D) \cap H$ of the system (1.1), that is, $u=\left(u_{1} \ldots, u_{n}\right) \in$ $C^{2}(\Omega, D) \cap H$ such that

$$
\begin{array}{r}
\int_{\Omega}[\Delta u \cdot \Delta \phi-c \nabla u \cdot \nabla \phi] d x-\int_{\Omega} \operatorname{grad}_{u} G(x, u(x)) \cdot \phi=0, \\
\quad \text { for all } \phi \in C^{2}(\Omega, D) \cap H .
\end{array}
$$

Let us introduce an open set of the Hilbert space $H$ as follows

$$
X=\left\{u \in H \mid u(x) \in D \subset R^{n}, x \in \Omega\right\} .
$$

Let us consider the functional on $X$

$$
\begin{aligned}
I(u) & =\frac{1}{2} \int_{\Omega}\left[\|\Delta u(x)\|_{R^{n}}^{2}-c\|\nabla u(x)\|_{R^{n}}^{2}\right] d x-\int_{\Omega} G(x, u) d x, \\
& =Q(u)-\int_{\Omega} G(x, u) d x \\
& =\frac{1}{2}\left\|P^{+} u\right\|^{2}-\frac{1}{2}\left\|P^{-} u\right\|^{2}-\int_{\Omega} G(x, u) d x d t
\end{aligned}
$$

where $Q(u)=\frac{1}{2} \int_{\Omega}\left[\|\Delta u(x)\|_{R^{n}}^{2}-c\|\nabla u(x)\|_{R^{n}}^{2}\right] d x$ and $\|u\|^{2}=\sum_{i=1}^{n}\left\|u_{i}\right\|^{2}$. The Euler equation for (2.1) is (1.1). By the following Lemma 2.1, $I \in C^{1}(X, R)$, and so the weak solutions of system (1.1) coincide with the critical points of the associated functional $I(u)$.

Lemma 2.1. Assume that $G$ satisfies the conditions $(G 1)-(G 2)$. Then $I(u)$ is continuous and Fréchet differentiable in $X$ with Fréchet derivative

$$
\begin{aligned}
& D I(u) v=\int_{\Omega}\left[\Delta u(x) \cdot \Delta v(x)-c \nabla u(x) \cdot \nabla v(x)-\operatorname{grad}_{u} G(x, u(x)) \cdot v(x)\right] d x \\
& \forall v \in X . \\
& \text { Moreover } D I \in C \text {. That is, } I \in C^{1} .
\end{aligned}
$$


Proof. First we prove that $I(u)$ is continuous. For $u, v \in X$,

$$
\begin{aligned}
& |I(u+v)-I(u)| \\
= & \mid \frac{1}{2} \int_{\Omega}\left(\Delta^{2}(u+v)+c \Delta(u+v)\right) \cdot(u+v) d x \\
& -\int_{\Omega} G(x, u+v) d x \\
& -\frac{1}{2} \int_{\Omega}\left(\Delta^{2} u+c \Delta u\right) \cdot u d x+\int_{\Omega} G(x, u) d x \\
= & \mid \frac{1}{2} \int_{\Omega}\left[\left(\Delta^{2} u+c \Delta u\right) \cdot v+\left(\Delta^{2} v+c \Delta v\right) \cdot u+\left(\Delta^{2} v+c \Delta v\right) \cdot v\right] d x \\
& -\int_{\Omega}(G(x, u+v)-G(x, u)) d x \mid .
\end{aligned}
$$

We have

$$
\begin{gathered}
\left|\int_{\Omega}[G(x, u+v)-G(x, u)] d x\right| \\
\leq\left|\int_{\Omega}\left[\operatorname{grad}_{u} G(x, u(x)) \cdot v+O\left(\|v\|_{R^{n}}\right)\right] d x\right|=O\left(\|v\|_{R^{n}}\right) .
\end{gathered}
$$

Thus we have

$$
|I(u+v)-I(u)|=O\left(\|v\|_{R^{n}}\right) .
$$

Next we shall prove that $I(u)$ is Fréchet differentiable in $X$. For $u, v \in$ $X$

$$
\begin{aligned}
& |I(u+v)-I(u)-D I(u) v| \\
= & \mid \frac{1}{2} \int_{\Omega}\left(\Delta^{2}(u+v)+c \Delta(u+v)\right) \cdot(u+v) d x-\int_{\Omega} G(x, u+v) d x \\
& -\frac{1}{2} \int_{\Omega}\left(\Delta^{2} u+c \Delta u\right) \cdot u d x+\int_{\Omega} G(x, u) d x \\
& -\int_{\Omega}\left(\Delta^{2} u+c \Delta u-\operatorname{grad}_{U} G(x, u(x))\right) \cdot v d x \mid \\
= & \mid \frac{1}{2} \int_{\Omega}\left[\left(\Delta^{2} v+c \Delta v\right) \cdot u+\left(\Delta^{2} v+c \Delta v\right) \cdot v\right] d x \\
& -\int_{\Omega}[G(x, u+v)-G(x, u)] d x+\int_{\Omega} \operatorname{grad}_{u} G(x, u(x)) \cdot v d x \mid .
\end{aligned}
$$

Thus by (2.3), we have

$$
|I(u+v)-I(u)-D I(u) v|=O\left(\|v\|_{R^{n}}\right) .
$$


Similarly, it is easily checked that $I \in C^{1}$.

Let

$$
X^{+}=X \cap H^{+} \quad X^{-}=X \cap H^{-} .
$$

Lemma 2.2. Assume that $\lambda_{j}<c<\lambda_{j+1}, j \geq 1$, and $G$ satisfies the conditions $(G 1)-(G 2)$. Let $\left\{u_{k}\right\} \subset X^{-}$and $u_{k} \rightarrow u$ weakly in $X$ with $u \in \partial X$. Then $I\left(u_{k}\right) \rightarrow-\infty$.

Proof. For the proof of the conclusion, it suffices to prove that

$$
\int_{\Omega} G\left(x, u_{k}(x)\right) d x \longrightarrow+\infty .
$$

Since $G(x, u(x))$ is bounded from below, it suffices to prove that there is a subset $\tilde{\Omega}$ of $\Omega$ such that

$$
\int_{\tilde{\Omega}} G\left(x, u_{k}(x)\right) d x \longrightarrow+\infty .
$$

$u \in \partial X$ means that there exists $x^{*} \in \Omega$ such that $u\left(x^{*}\right) \in \partial D$. Let us set

$$
\Omega_{\delta}\left(x^{*}\right)=\left\{x \in \Omega \mid\left\|x-x^{*}\right\|_{R^{n}}<\delta\right\} .
$$

By $(G 1)$ and $(G 2)$, there exists a constant $B$ such that

$$
G(x, u) \geq \frac{A}{d^{2}(u, C)}-B
$$

Thus we have

$$
\int_{\Omega_{\delta}\left(x^{*}\right)} G(x, u(x)) d x \geq \int_{\Omega_{\delta}\left(x^{*}\right)}\left(\frac{A}{\left\|u(x)-u\left(x^{*}\right)\right\|_{R^{n}}^{2}}-B\right) d x
$$

for all $\delta>0$. By Schwarz's inequality, we have

$$
\left\|u(x)-u\left(x^{*}\right)\right\|_{R^{n}} \leq\left\|x-x^{*}\right\|_{R^{n}}^{\frac{1}{2}}\left(\int_{\Omega}\|\nabla u(x)\|_{R^{n}}^{2}\right)^{\frac{1}{2}} \leq \delta^{\frac{1}{2}}\left(\int_{\Omega}\|\nabla u(x)\|_{R^{n}}^{2}\right)^{\frac{1}{2}} .
$$

Thus we have

$$
\int_{\Omega_{\delta}\left(x^{*}\right)} G(x, u(x)) d x \geq \int_{\Omega_{\delta}\left(x^{*}\right)}\left(\frac{A}{\delta \int_{\Omega}\|\nabla u(x)\|_{R^{n}}^{2}}-B\right) d x \longrightarrow \infty .
$$

Hence

$$
\int_{\Omega_{\delta}\left(x^{*}\right)} G(x, u(x)) d x=\infty .
$$

Since the embedding $H \hookrightarrow C\left(\Omega, R^{n}\right)$ is compact, we have

$$
\max \left\{\left\|u(x)-u_{k}(x)\right\|_{R^{n}}^{2} \mid x \in \Omega\right\} \longrightarrow 0 \quad \text { as } k \rightarrow \infty .
$$


Thus by Fatou's lemma, we have

$$
\begin{gathered}
\liminf \int_{G_{\delta}\left(x^{*}\right)} G\left(x, u_{k}(x)\right) \geq \int_{G_{\delta}\left(x^{*}\right)} \liminf G\left(x, u_{k}(x)\right) \\
=\int_{G_{\delta}\left(x^{*}\right)} G(x, u(x))=+\infty
\end{gathered}
$$

Thus

$$
\liminf \int_{G_{\delta}\left(x^{*}\right)} G\left(x, u_{k}(x)\right)=+\infty .
$$

Thus for $u_{k} \in X^{-}$,

$$
\begin{aligned}
I\left(u_{k}\right) & =\int_{\Omega}\left[\frac{1}{2}\|\Delta u(x)\|_{R^{n}}^{2}-c\|\nabla u(x)\|_{R^{n}}^{2}-G\left(x, u_{k}(x)\right)\right] d x \\
& =\frac{1}{2}\left\|P^{+} u_{k}\right\|^{2}-\frac{1}{2}\left\|P^{-} u_{k}\right\|^{2}-\int_{\Omega} G\left(x, u_{k}(x)\right) d x \\
& =-\frac{1}{2}\left\|P^{-} u_{k}\right\|^{2}-\int_{\Omega} G\left(x, u_{k}(x)\right) d x \rightarrow-\infty,
\end{aligned}
$$

so we prove the lemma.

Now we recall the generalized mountain pass theorem (cf. Theorem 5.3 in $[8])$.

Let

$$
\begin{gathered}
B_{r}=\{u \in X \mid\|u\| \leq r\}, \\
\partial B_{r}=\{u \in X \mid\|u\|=r\} .
\end{gathered}
$$

TheOREM 2.1. (Generalized mountain pass theorem)

Let $X$ be a real Banach space with $X=V \oplus W$, where $V \neq\{0\}$ and is finite dimensional. Suppose that $I \in C^{1}(X, R)$, satisfies (P.S.) condition, and

(i) there are constants $\rho, \alpha>0$ and a bounded neighborhood $B_{\rho}$ of 0 such that $\left.I\right|_{\partial B_{\rho} \cap W} \geq \alpha$, and

(ii) there is an $e \in \partial B_{1} \cap W$ and $R>\rho$ such that if $K=\left(\bar{B}_{R} \cap V\right) \oplus$ $\{$ re $\mid 0<r<R\}$, then $\left.I\right|_{\partial K} \leq 0$.

Then $I$ possesses a critical value $b \geq \alpha$. Moreover $b$ can be characterized as

$$
b=\inf _{\gamma \in \Gamma} \max _{u \in K} I(\gamma(u)),
$$

where

$$
\Gamma=\{\gamma \in C(\bar{K}, X) \mid \gamma=i d \text { on } \partial K\}
$$




\section{Proof of Theorem 1.1}

We shall show that the functional $I(u)$ satisfies the geometric assumptions of the generalized mountain pass theorem.

LEMMA 3.1. (Palais-Smale condition)

Assume that $\lambda_{j}<c<\lambda_{j+1}, j \geq 1$, and $G$ satisfies the conditions (G1) and $(G 2)$. Then $I(u)$ satisfies the (P.S.) condition in $X$.

Proof. We shall prove the lemma by contradiction. We suppose that there exists a sequence $\left\{u_{k}\right\} \subset X$ satisfying $I\left(u_{k}\right) \rightarrow \gamma$ and

$$
D I\left(u_{k}\right)=\Delta^{2} u_{k}+c \Delta u_{k}-\operatorname{grad}_{u} G\left(x, u_{k}(x)\right) \longrightarrow \theta \quad \text { in } X,
$$

or equivalently

$$
u_{k}-\left(\Delta^{2}+c \Delta\right)^{-1}\left(\operatorname{grad}_{u} G\left(x, u_{k}(x)\right)\right) \longrightarrow \theta,
$$

where $\theta=(0, \cdots, 0)$ and $\left(\Delta^{2}+c \Delta\right)^{-1}$ is a compact operator. We claim that the sequence $\left\{u_{k}\right\}$, up to a subsequence, converges. It suffices to prove that the sequence $\left\{u_{k}\right\}$ is bounded in $X$. By contradiction, we suppose that $\left\|u_{k}\right\|_{R^{n}} \rightarrow \infty$. Then for large $k$, we have

$$
\left\|u_{k}\right\|_{R^{n}} \geq R_{0} \text {. }
$$

It follows from (3.2) that

$$
\left|\int_{\Omega} G\left(x, u_{k}\right) d x\right| \leq|\Omega| \sup \left\{\left|G\left(x, u_{k}\right)\right| \mid\left(x, u_{k}\right) \in \Omega \times\left(R^{n} \backslash B_{R_{0}}\right)\right\} .
$$

Let us set $w_{k}=\frac{u_{k}}{\left\|u_{k}\right\|}$. Then $\left\|w_{k}\right\|=1$, and hence the subsequence $\left\{w_{k}\right\}$, up to a subsequence, converges weakly to $w$ with $\|w\|=1$. By (3.1), we have

$$
\begin{aligned}
0 \longleftarrow \frac{D I\left(u_{k}\right) u_{k}}{\left\|u_{k}\right\|_{H}} & =\int_{\Omega}\left(\Delta^{2} w_{k}+c \Delta w_{k}\right) \cdot w_{k} d x-\int_{\Omega} \frac{G\left(x, u_{k}\right)}{\left\|u_{k}\right\|^{2}} \\
& =\left\|P^{+} w_{k}\right\|^{2}-\left\|P^{-} w_{k}\right\|^{2}-\int_{\Omega} \frac{G\left(x, u_{k}\right)}{\left\|u_{k}\right\|^{2}} .
\end{aligned}
$$

Letting $k \rightarrow \infty$ in (3.4), by (3.3), we have

$$
\begin{aligned}
0 & =\lim _{k \rightarrow \infty}\left\|P^{+} w_{k}\right\|^{2}-\lim _{k \rightarrow \infty}\left\|P^{-} w_{k}\right\|^{2} \\
& =\int_{\Omega}\left(\Delta^{2} w+c \Delta w\right) \cdot w d x \\
& =\left\|P^{+} w\right\|^{2}-\left\|P^{-} w\right\|^{2} .
\end{aligned}
$$


Thus we have

$$
\lim _{k \rightarrow \infty}\left\|P^{+} w_{k}\right\|^{2}=\left\|P^{+} w\right\|^{2}, \quad \lim _{k \rightarrow \infty}\left\|P^{-} w_{k}\right\|^{2}=\left\|P^{-} w\right\|^{2} .
$$

Thus

$$
\lim _{k \rightarrow \infty}\left\|w_{k}\right\|=\|w\|
$$

and by (3.5), $w$ is the weak solution of the equation

$$
\Delta^{2} w+c \Delta w=0 \quad \text { in } X .
$$

Since $c$ is not the eigenvalue, $w=(0, \cdots, 0)$ is the only weak solution of (3.6), which is absurd to the fact that $\|w\|=1$. Thus $\left\{u_{k}\right\}$ is bounded. Thus the subsequence, up to a subsequence, $u_{k}$ converges weakly to $u$ in $X$. By Lemma 2.2, $u \in X$ and that $\left\|\operatorname{grad}_{u} G\left(\cdot, u_{k}\right)\right\|$ is bounded. Since $\left(\Delta^{2}+c \Delta\right)^{-1}$ is compact and (3.1) holds, $\left\{u_{k}\right\}$ converges strongly to $u$. Thus we prove the lemma.

Let

$$
K=\left(\bar{B}_{r} \cap X^{-}\right) \oplus\left\{r e \mid e \in B_{1} \cap X^{+}, 0<r<R\right\} .
$$

Lemma 3.2. Assume that $\lambda_{j}<c<\lambda_{j+1}, j \geq 1$, and $G$ satisfies the conditions $(G 1)$ and $(G 2)$. Then there exist sets $S_{\rho} \subset X^{+}$with radius $\rho>0, K \subset X$ and constants $\alpha>0$ such that

(i) $S_{\rho} \subset X^{+}$and $\left.I\right|_{S_{\rho}} \geq \alpha$,

(ii) $K$ is bounded and $\left.I\right|_{\partial K} \leq 0$,

(iii) $S_{\rho}$ and $\partial K$ link.

Proof. (i) Let us choose $u \in X^{+} \subset X$. Then $u(x) \in D$. By (G1), $G(x, u)$ is bounded above and there exists a constant $C>0$

$$
I(u)=\frac{1}{2}\left\|P^{+} u\right\|^{2}-\frac{1}{2}\left\|P^{-} u\right\|^{2}-\int_{\Omega} G(x, u) d x \geq \frac{1}{2}\left\|P^{+} u\right\|^{2}-C
$$

for $C>0$. Then there exist a constant $\rho>0$ and $\alpha>0$ such that if $u \in S_{\rho} \cap X^{+}$, then $I(u) \geq \alpha$.

(ii) Let us choose $e \in B_{1} \cap X^{+}$. Let $u \in \bar{B}_{r} \cap X^{-} \oplus\{r e \mid 0<r\}$. Then $u=v+w, v \in \bar{B}_{r} \cap X^{-}, w=r e$. We note that

$$
\text { If } v \in \bar{B}_{r} \cap X^{-} \text {, then } \int_{\Omega}\left[\|\Delta v(x)\|_{R^{n}}^{2}-c\left\|\nabla v_{x}\right\|_{R^{n}}^{2}\right] d x=-\left\|P^{-} u\right\|^{2} \leq 0 \text {. }
$$

By (G2), $G(x, v+r e)$ is bounded from below. Thus by Lemma 2.2, there exists a constant $A>0$ such that if $u=v+r e$, then we have

$$
I(u)=\frac{1}{2} r^{2}-\frac{1}{2}\left\|P^{-} v\right\|^{2}-\int_{\Omega} G(x, v+r e) d x .
$$




$$
\leq \frac{1}{2} r^{2}-\frac{1}{2}\left\|P^{-} v\right\|^{2}-\int_{\Omega} \frac{A}{d^{2}(v+r e, C)} d x .
$$

We can choose a constant $R>r$ such that if $u=v+r e \in K=$ $\left(\bar{B}_{r} \cap X^{-}\right) \oplus\left\{r e \mid e \in B_{1} \cap X^{+}, 0<r<R\right\}$, then $I(u)<0$. Thus we prove the lemma.

By Lemma 2.1, $I(u)$ is continuous and Fréchet differentiable in $X$ and moreover $D I \in C$. By Lemma 2.2, If $\left\{u_{k}\right\} \subset X^{-}$and $u_{k} \rightarrow u$ weakly in $X$ with $u \in \partial X$, then $I\left(u_{k}\right) \rightarrow-\infty$. By Lemma 3.1, $I(u)$ satisfies the (P.S.) condition. By Lemma 3.2, there exist sets $S_{\rho} \subset X^{+}$ with radius $\rho>0, K \subset X$ and constants $\alpha>0$ such that $\left.I\right|_{S_{\rho}} \geq \alpha, K$ is bounded and $\left.I\right|_{\partial K} \leq 0$, and $S_{\rho}$ and $\partial K$ link. By the critical point theorem, $I(u)$ possesses a critical value $c \geq \alpha$. Thus (1.1) has at least one nontrivial weak solution. Thus we prove Theorem 1.1

\section{References}

[1] K.C. Chang, Infinite dimensional Morse theory and multiple solution problems, Birkhäuser, (1993).

[2] Q. H. Choi and T. Jung, Multiplicity of solutions and source terms in a fourth order nonlinear elliptic equation, Acta Math. Sin. 19 (4) (1999), 361-374.

[3] Q. H. Choi and T. Jung, Multiplicity results on nonlinear biharmonic operator, Rocky Mountain J. Math. 29 (1) (1999), 141-164.

[4] T. Jung and Q. H. Choi, Nonlinear biharmonic problem with variable coefficient exponential growth term, Korean J. Math. 18 (3) (2010), 1-12.

[5] T. Jung and Q. H. Choi, Multiplicity results on a nonlinear biharmonic equation, Nonlinear Anal. 30 (8) (1997), 5083-5092.

[6] T. Jung and Q. H. Choi, Nontrivial solution for the biharmonic boundary value problem with some nonlinear term, Korean J. Math., to be appeared (2013).

[7] A. M. Micheletti and A. Pistoia, Multiplicity results for a fourth-order semilinear elliptic problem, Nonlinear Anal. 31 (7) (1998), 895-908.

[8] P. H. Rabinowitz, Minimax methods in critical point theory with applications to differential equations, CBMS Reg. Conf. Ser. Math. 65, Amer. Math. Soc., Providence, Rhode Island (1986).

[9] Tarantello, A note on a semilinear elliptic problem, Differential Integral Equations 5 (3) (1992), 561-565. 
Department of Mathematics

Kunsan National University

Kunsan 573-701, Korea

E-mail: tsjung@kunsan.ac.kr

Department of Mathematics Education

Inha University

Incheon 402-751, Korea

E-mail: qheung@inha.ac.kr 